A

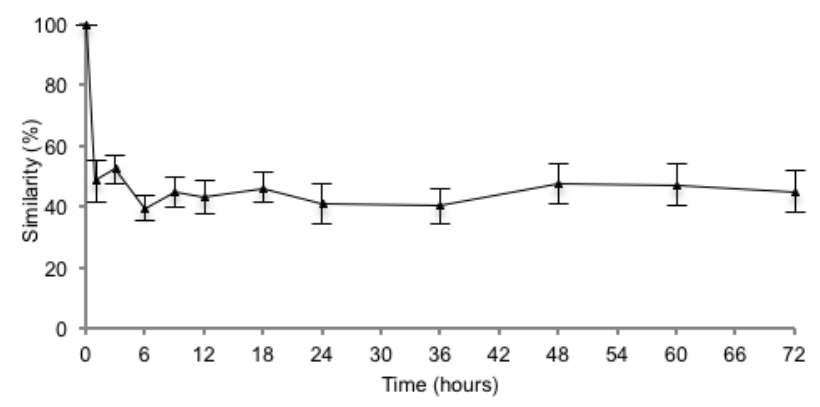

B

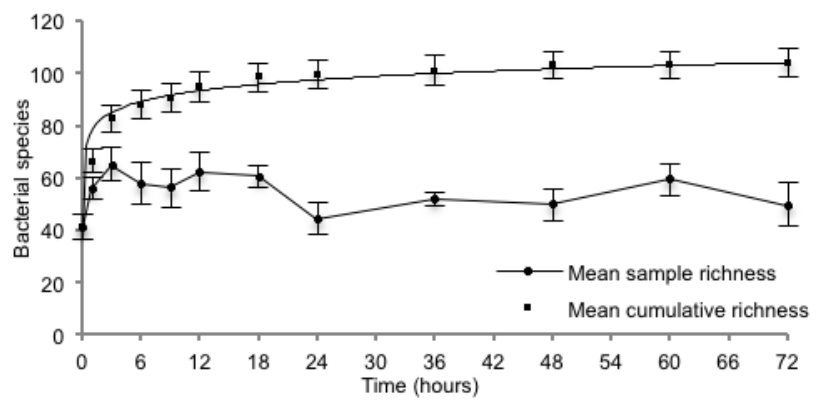

Abstract P89 Figure 1 (A) Change in bacterial community composition and abundance over 72 hours, the sample at each time point was compared to the sample frozen immediately. (B) Mean cumulative richness (emerging new bacterial species) and mean sample richness (total number of bacterial species in each sample). Error bars represent the standard error of the mean.

(NCFBr). We assessed the impact of factors associated with NCFBr disease severity on bone mineral density $(\mathrm{BMD})$ and DEXA referral patterns in NCFBr.

Methods BMD reports from DEXA scanners were collected for all $\mathrm{NCFBr}$ patients from our specialist clinic database and T-scores for lumbar spine (LS), total left hip (TLH) and neck of femur (NOF) were recorded. Osteoporosis was defined as $\mathrm{T}$-scores $\leq-2.5$, osteopenia between -1 and -2.5 and normal $B M D \geq-1$. FEV 1 values, exacerbation frequency and Pseudomonas aeruginosa chronic infection (defined as $>1$ positive sputum result in past 12 months) were recorded.

Results $336 \mathrm{NCFBr}$ patients were identified attending the specialist clinic. 101 patients had DEXA scans performed (30\%); 96 reports were retrievable. The male:female ratio was significantly different between scanned patients (29\%:71\%) and the entire NCFBr patient cohort (41\%:59\%): $p<0.01$ (two-tailed chi-squared test). Osteoporosis was detected in the same percentage of scanned males as females (32\%, any site). The prevalence of osteoporosis identified was similar across different scan sites (see table). Mean FEV (\% predicted) for DEXA-scanned patients was 58.2 ( \pm 28.4 ), 24\% were colonised with $P$. aeruginos $a$ and mean number of exacerbations was $4.7 /$ year $( \pm 3.0 ; n=44)$. No significant correlation was found between $\mathrm{T}$-scores (any site) and $\mathrm{FEV}_{1} \%$ pred values, $P$. aeruginosa colonisation or number of exacerbations.

Conclusion More than $40 \%$ of NCFBr patients have osteopenia and more than 25\% have osteoporosis; this is higher than seen in COPD (e.g. NHANES, Schnell et al, 2012). Male patients had lower DEXA scanning rates suggesting referral bias favouring females. Low BMD is not predictable based on $\mathrm{NCFBr}$ "disease severity parameters". Greater emphasis on investigating NCFBr patients for osteoporosis, particularly males, may improve the incidence rate of fragility fractures in this patient population. Future studies on steroid use and osteoporosis incidence rates in this population would also be beneficial.
Abstract P90 Table 1 NCFBr patients with osteoporosis, osteopenia or normal bone mineral density measurements at different DEXA scan sites

\begin{tabular}{lccc}
\hline & \multicolumn{3}{c}{ BMD classification } \\
\cline { 2 - 4 } Scanning site & Normal (\%) & 0steopenia (\%) & 0steoporosis (\%) \\
\hline Lumbar spine & $28(29)$ & $44(46)$ & $24(25)$ \\
Total Left Hip & $32(34)$ & $38(40)$ & $25(26)$ \\
Neck of Femur & $22(24)$ & $43(47)$ & $27(29)$ \\
\hline
\end{tabular}

\section{P91 ANTI-IGE THERAPY: AN OBSERVATION IN CYSTIC FIBROSIS}

doi:10.1136/thoraxjnl-2012-202678.333

IE Robinson, JB Morjaria, T Moon, I Molyneux, AH Morice. Cardiovascular and Respiratory Studies, Hull York Medical School, University of Hull, Castle Hill Hospital, Hull, UK

Introduction Cystic Fibrosis (CF) is characterised by the development of progressive cystic bronchiectasis. A proportion of patients have asthmatic elements which respond to conventional asthma therapy. Omalizumab (Xolair®, Novartis, UK) is a recombinant humanised monoclonal IgG which binds to IgE. We hypothesise that an IgE mediated component of airway inflammation in $\mathrm{CF}$ may respond to omalizumab.

Methods Seven patients mean age 31 (SD \pm 12 ) years, serum IgE (30-1500), symptomatic despite maximal conventional therapy were administered omalizumab using standard dosing regimen in an open label fashion. The Asthma Control Test (ACT), Asthma Quality of Life Questionnaire (AOLO) and FEV were recorded at baseline and sixteen weeks post-treatment. Days of intravenous (IV) antibiotic in the year before and during therapy were compared.

Results There was a significant improvement in the mean ACT score from $11( \pm 3.7)$ to $17( \pm 5.9), p=0.031$. AOLO scores pre-omalizumab $49( \pm 15)$ vs. post-omalizumab $69( \pm 22), p=0.156$; and $\% \mathrm{FEV}_{1}$ pre-omalizumab $42 \%( \pm 13 \%)$ vs. post-omalizumab $45 \%$ $( \pm 14 \%), p=0.078$ were not significantly improved. Similarly, the number of days of IV antibiotic usage declined with omalizumab treatment from 43 days ( \pm 24 days) to 25 days ( \pm 16 days) $p=0.297$ respectively.

Discussion This small study shows an improvement in symptom control in selected CF patients with omalizumab and other indices showed non-significant improvements, particularly IV antibiotic usage. Previous studies have demonstrated a correlation between exacerbation frequency in CF patients and the rate of decline in lung function. These findings warrant larger trials of omalizumab in 'asthmatic' CF

\section{P92 A RETROSPECTIVE STUDY TO EVALUATE THE USE OF NEBULISED MEROPENEM AT A LARGE UK ADULT CF CENTRE}

doi:10.1136/thoraxjnl-2012-202678.334

V Carrolan, EF Nash, R Rashid, JL Whitehouse. West Midlands Regional Adult CF Centre, Heart of England NHS Foundation Trust, Birmingham, United Kingdom

Background Meropenem is commonly used intravenously in CF patients infected with Pseudomonas aeruginosa, but there is limited evidence of its tolerability and effectiveness when used as a nebulised treatment.

Aim To evaluate the use of nebulised meropenem in our large UK adult CF centre.

Methods Medical records of patients who had trialled nebulised meropenem between 2008-2012 were reviewed for: demographics, lung function, BMI, sputum microbiology, indications and tolerability and number of exacerbations requiring IV antibiotics. 
Results 37 patients (18 male) trialled nebulised meropenem, with median age (IOR) 27 (20-37) years and $\mathrm{FEV}_{1} \%$ predicted $48 \%$ (35-64\%). Patients received a $250 \mathrm{mg}$ dose reconstituted with water for injection preceded by a bronchodilator. All patients were chronically infected with $P$. aeruginosa. The most common treatment indications were deteriorating condition or intolerance to other antibiotic nebulisers (21/37), with additional indications including treatment of Burkholderia cepacia complex (8/37) and non-tuberculous mycobacteria (8/37). 22/37 continue to tolerate the drug well. 10 of these 22 had previously been intolerant to Tobramycin Inhalation Solution (TIS) and 1 intolerant to nebulised amikacin. 4/37 discontinued due to no benefit or poor concordance. 11/37 did not tolerate nebulised meropenem, reporting adverse effects including chest tightness, increased cough, chest discomfort, nausea and lethargy. 6 of these 11 had previously been intolerant to TIS and 2 were intolerant to amikacin. There were no significant changes in lung function, $\mathrm{BMI}$ or requirement for IV antibiotics comparing the 12-month periods before and after commencing nebulised meropenem.

Conclusion Nebulised meropenem is generally well tolerated in CF adults and offers an alternative antibiotic choice for people with CF unable to tolerate other treatments.

\section{Abstract P92 Table 1}

\begin{tabular}{lcccc}
\hline & $\begin{array}{l}\text { No. courses of } \\
\text { IVs 12/12 pre } \\
\text { mero }\end{array}$ & $\begin{array}{l}\text { No. courses of } \\
\text { IVs 12/12 post } \\
\text { mero }\end{array}$ & $\begin{array}{l}\text { Days in hosp } \\
\text { 12/12 pre } \\
\text { mero }\end{array}$ & $\begin{array}{l}\text { Days in hosp } \\
\text { 12/12 post } \\
\text { mero }\end{array}$ \\
\hline M. abscessus & 7 & 10 & 43 & 44 \\
B. cepacia complex & 8 & 5 & 43 & 11 \\
Other & 23 & 25 & 211 & 293 \\
\hline
\end{tabular}

\section{P93 CLINICAL OUTCOMES AND PATIENT SATISFACTION FOLLOWING INITIATION OF THE TOBI PODHALER IN CF ADULTS}

doi:10.1136/thoraxjnl-2012-202678.335

CJ Brown, EF Nash, V Carrolan, R Rashid, JL Whitehouse. West Midlands Adult CF Centre, Heart of England NHS Foundation Trust, Birmingham, United Kingdom

Background In the clinical trial setting, TOBI Podhaler (TIP) has been shown to be non-inferior to nebulised Tobramycin Inhalation Solution (TIS) in CF subjects in terms of effects on lung function, with increased adverse events but overall superior patient satisfaction. However, there is currently little 'real world' evidence of tolerance and effectiveness of TIP.

Aim To assess the experience of CF adults receiving TIP at our large regional UK adult CF centre.

Methods We report the preliminary results of a prospective observational study to evaluate changes in lung function, tolerability and antibiotic requirements of $73 \mathrm{CF}$ adults that have received at least one dose of TIP at our centre to date. Patients also completed a visual analogue scale (VAS) score of tolerance, convenience, satisfaction and likelihood to continue ( $1=$ very satisfied/not at all severe/ very likely, $10=$ not at all satisfied/very severe/not at all likely) at their 28-day review.

Results Adverse events (AEs) were common following the test dose (most commonly cough in $24 / 73,32.9 \%$ ), although only 1 patient discontinued treatment, due to a 10\% drop in FEV1\% predicted. $27 / 72$ patients so far have completed 28 days treatment, with median change in FEV1\% predicted of $-1.7 \%$ (IOR -6.1 to 2.4\%). 12/27 patients (44.4\%) reported AEs at their 28-day review; most commonly cough (10 patients, $37.0 \%$ ), with median VAS score for severity of all AEs of 4/10. $12 / 27$ patients (44.4\%) were commenced on oral or IV antibiotics for pulmonary exacerbation at their 28-day review. Median VAS scores for overall satisfaction, ease of administration, and likelihood to continue were all 1/10. 12/72 patients to date have discontinued TIP before completion (10 patients) or at completion ( 2 patients) of 28 days treatment, 8 $(66.7 \%)$ of whom were previously intolerant of TIS. The most common reason for discontinuation was cough (9/12 patients, $75.0 \%)$, with median VAS score for severity of all AEs $7 / 10$.

Conclusion Despite experiencing a high incidence of adverse events, patients commencing TIP report high levels of treatment satisfaction.

\section{P94 SHOULD ADRENALINE AUTO-INJECTORS BE PRESCRIBED FOR CYSTIC FIBROSIS PATIENTS HAVING HOME INTRAVENOUS ANTIBIOTICS? - A UK NATIONAL AUDIT}

doi:10.1136/thoraxjnl-2012-202678.336

${ }^{1}$ BN Fernandes, ${ }^{2} \mathrm{~A}$ Jones, ${ }^{1} \mathrm{~A}$ Simpson, ${ }^{2} \mathrm{~K}$ Webb, ${ }^{1} \mathrm{~A}$ Custovic. ${ }^{1}$ The Allergy Centre, University Hospital of South Manchester, Manchester, UK; ${ }^{2}$ Manchester Adult Cystic Fibrosis Centre, University Hospital of South Manchester, Manchester, UK

Introduction The UK Cystic Fibrosis (CF) Trust antibiotic guidelines recommends that all patients having home intravenous antibiotics (HIVA) should have an "anaphylactic kit" at home. However, the practise of prescribing adrenaline auto-injectors (AAI) for patients having HIVA varies widely across the country. Studies on HIVA have shown that they are safe, especially if first dose is given in hospital. The aim of this audit is to assess compliance with CF Trust antibiotic guidelines and discover how many patients have experienced anaphylaxis at home while on HIVA.

Methods The audit was performed online from April to September 2011 by asking respondents to fill in a web-based questionnaire. An email was sent to 59 adult and paediatric UK CF centre leads requesting them to take part in this audit.

Results 55/59 (93\%) took part in the audit. 16 (29\%) routinely prescribe AAI whereas others do not or only prescribe in patients with previous history of allergic reactions to antibiotics (Figure 1). 5 physicians reported they knew patients who had experienced anaphylaxis at home. These physicians reported a total of at least 10 anaphylaxis episodes in the last 5 years. 1 mentioned they occurred after the 1 st dose, 3 after the 3 rd dose and 2 were unsure. 3 reported these patients used their AAI and 3 did not. We estimate that if the average annual number of HIVA courses is 100 courses per centre, this equates to approximately 1 anaphylaxis episode per 2,700 courses of HIVA.

Conclusions Most CF centres do not routinely prescribe AAI, in contrast to the guidelines issued by the CF trust. Anaphylactic reactions do occur in CF patients having HIVA but this is a relatively rare event. However, as this is a retrospective study based on recall of previous events, the precise nature of the reported reactions cannot be confirmed, so the true prevalence of anaphylactic reactions related to HIVA cannot be ascertained. A prospective multicentre study recording reactions to HIVA and evaluating them in detail (preferably by an allergist) is required.

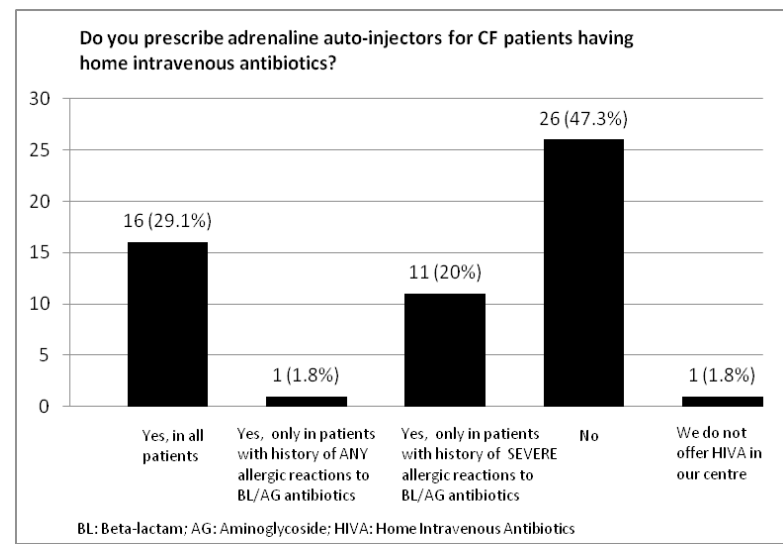

Abstract P94 Figure 